\title{
Coronal loops, flare ribbons and aurora during slip-running
}

\author{
Brigitte Schmieder ${ }^{1}$, Guillaume Aulanier ${ }^{1}$, Pascal Démoulin ${ }^{1}$, and Etienne Pariat $^{2}$ \\ ${ }^{1}$ Observatoire de Paris, LESIA, 92195 Meudon, France \\ ${ }^{2} N R L$, Washington DC, USA
}

(Received September 12, 2007; Accepted August 16, 2008; Online published May 29, 2009)

\begin{abstract}
Solar two ribbon flares are commonly explained by magnetic field reconnections in the low corona. During the reconnection energetic particles (electrons and protons) are accelerated from the reconnection site. These particles are following the magnetic field lines down to the chromosphere. As the plasma density is higher in these lower layers, there are collisions and emission of radiation. Thus bright ribbons are observed at both ends of flare loops. These ribbons are typically observed in $\mathrm{H} \alpha$ and in EUV with SoHO and TRACE. As the time is going, these ribbons are expanding away of each other. In most studied models, the reconnection site is a separator line, where two magnetic separatrices intersect. They define four distinct connectivity domains, across which the magnetic connectivity changes discontinuously. In this paper, we present a generalization of this model to $3 \mathrm{D}$ complex magnetic topologies where there are no null points, but quasi-separatrices layers instead. In that case, while the ribbons spread away during reconnection, we show that magnetic field lines can quickly slip along them. We propose that this new phenomenon could explain fast extension of H $\alpha$ and TRACE $1600 \AA$ ribbons, fast moving HXR footpoints along the ribbons as observed by RHESSI, and that it is observed in soft X rays with Hinode/XRT.
\end{abstract}

Key words: Sun: flares, magnetosphere: aurora.

\section{Introduction}

Eruptions occur at locations on the Sun where there is a build-up of a large amount of magnetic energy and when this energy is released via some reconnection process. Many models deal with how this energy release is accomplished; for example Forbes and Isenberg (1991), Lin et al. (1998) discuss on the convergence of magnetic flux below a flux tube situated along a magnetic polarity inversion line that leads to enhancement of the flux tube and eruption. Chen and Shibata (2000) presents a model where eruption results when the tension of the sheared arcade or flux tube field is weakened via reconnection between the main magnetic field and emerging flux (kind of "tether-cutting" mechanism). Antiochos et al. (1999) discuss instead disruption of field lines and reconnection above flux tubes. To determine which of the mechanism is predominantly responsible for eruptions it is necessary to observe the onset and the evolution of flares. The coronal plasma is frozen into the magnetic field almost everywhere except where current sheets can be formed and then dissipated. Current sheets develop along separatrices when the magnetic configuration evolves quasi-statically or dynamically. A very powerful tool to understand where the energy could be deposited is to study the magnetic topology of the active region, since it defines where magnetic reconnection is expected to occur (see reviews of Démoulin, 2005; Longcope, 2005; Schmieder, 2006).

In the first section, magnetic topology models are pre-

Copyright (c) The Society of Geomagnetism and Earth, Planetary and Space Sciences (SGEPSS); The Seismological Society of Japan; The Volcanological Society of Japan; The Geodetic Society of Japan; The Japanese Society for Planetary Sciences; TERRAPUB. sented. In the second and third sections, we review eruptive and compact flares showing that their dynamics cannot be neglected to interpret the formation of long ribbons with evolving $\mathrm{H} \alpha$, UV brightenings and X-ray kernels. New simulations are briefly described. Slip-running reconnection simulation explains the fast motion of X-ray sources along flare.

\section{Magnetic Topological Models}

Coronal activities such as flares, eruptions and general heating are often attributed to the manner in which the coronal field responds to photospheric motion. The coronal plasma has a low resistivity and to release efficiently the free energy, small length scales have to be created. Coronal magnetic fields are forced to evolve continuously by slow photospheric velocities $(0.1 \mathrm{~km} / \mathrm{s})$, faster velocities still lower to the typical Alfvén velocity $(\sim 1000 \mathrm{~km} / \mathrm{s})$ may exist during magnetic flux emergence. In this context magnetic configurations, with a slow evolution at the boundary leads to the formation of very thin current layers play a key role (review of Démoulin, 2006). Let us recall some classical definitions of magnetic configuration and reconnection. Separatrices are magnetic surfaces where the magnetic field line linkage is discontinuous, thus where the field lines can change abruptly of connectivity. In 2D configuration the reconnection occurs at a X-point (null point) where the magnetic vanishes, in 3D configuration the intersection of the field lines occur along the separator, intersection of the separatrices. Current sheet form along the whole separatrices when shearing flows are present in the photosphere. The flare ribbons are found at the intersections of the separatrices and the chromosphere. 
Flare ribbons and loops evidence the locations where the stored energy is released. For testing the spatial localization of the energy release sites, it is needed to extrapolate the field lines in the corona using photospheric magnetograms as a boundary condition. When the magnetic field is no more represented by point charges but maintained continuously as observed, there are not necessarily separatrices to interpret the ribbons: a generalization of the concept of separatrices does provide an interpretation of 3D magnetic reconnection (Démoulin et al., 1996a, 1997). This new concept is based on the existence of quasi-separatrice layers (QSL) for general magnetic fields anchored to a boundary. QSLs are defined as volumes where magnetic field lines can change of connectivity abruptly. There are no coronal null points in such configurations. Such topological properties are insensitive to detailed geometry of the magnetic field, and thereby create a very robust tool to understand the loci of flare energy release. The intersections of QSLs and the chromosphere are restricted to smaller regions than in the case of separatrices and match better the shape of the ribbons (Schmieder et al., 1997).

\section{Eruptive Flares}

Firstly we investigated the regions of large flares, we called eruptive flares when a coronal mass ejection (CME) is related to the flare. In this frame, Chen and Shibata (2000) and Lin (2004) proposed unified models. As the magnetic field lines are reconnected, a plasmoid is escaping and forming the CME. During the reconnection, electrons are precipiced downwards to denser layers. They heat the plasma and form the so-called two bright ribbons in the chromosphere (visible in $\mathrm{H} \alpha$, in $\mathrm{Ca}$ II $\mathrm{H}$ by Hinode/SOT, the $1600 \AA$ continuum by TRACE), in the transition region (CDS, EIT and TRACE). Between the ribbons, loops are observed in different wavelengths: bright $\mathrm{X}$ ray loops, in coronal lines $171 \AA$ and $195 \AA$ and bright or dark in $\mathrm{H} \alpha$ as they are cooling (Schmieder et al., 1996; DelZanna et al., 2006a). The loops form growing arcades with higher and higher hot loops while the reconnection point is rising. In the ribbons, kernels are the footpoints of loops (DelZanna et al., 2006b). It was shown that the later arcades are less and less sheared (Su et al., 2006). During cooling process the two ribbon distance is increasing. In complex active regions different systems of ribbons can be formed and developed in a fast time, of the order of few minutes (14 July 2000, 28 October 2003, 13 September 2005, 27 May 2003). In the event of 13 September 2005 a series of 10 flares occur within one day and even a series of 3 flares (X1.4, X 1.5, $\mathrm{X} 1.7)$ in three hours. The concerned active regions are all complex containing a spot with constant new emerging field with fast moving polarities. It is difficult to identify the trigger of each flare of the series.

Nevertheless, we are able to find the locations where the magnetic reconnection is possible and produce flares by analyzing the topology either by lfff (linear-force-free field) extrapolation when the shear is weak enough, otherwise by nlfff (non linear-force-free field) methods of extrapolation have to be applied (Régnier and Canfield, 2007).

Many models of flare onset are developed based on tether-cutting or straining forces (Klimchuk, 2001). For large quadrupolar configuration, the breakout model proposed by Antiochos (1999) seems to be successful for some cases (Aulanier et al., 2000). The existence of null point which was pointed out in the Antiochos model does not seem to be a necessary condition for breaking the overlying lines (Schmieder et al., 2006; Mandrini et al., 2006). Reconnection could occur in quasi separatrices layers with no null points (Li et al., 2006; Schmieder et al., 2007).

\section{QSL Reconnection with Slippage of Magnetic Field Lines}

In the previous section we have shown complex examples of flares with multiple ribbons formed at different places successively. Here we present a simple compact flare with no CME in order to study in details the dynamics of the ribbons, not the well known increasing distance of the ribbons versus time but the fast lateral extension of the ribbons. The flare occurs in a sheared active region at the location of a small emerging flux (Berlicki et al., 2004). The observation in $\mathrm{H} \alpha$ shows two ribbons, one of them is relatively
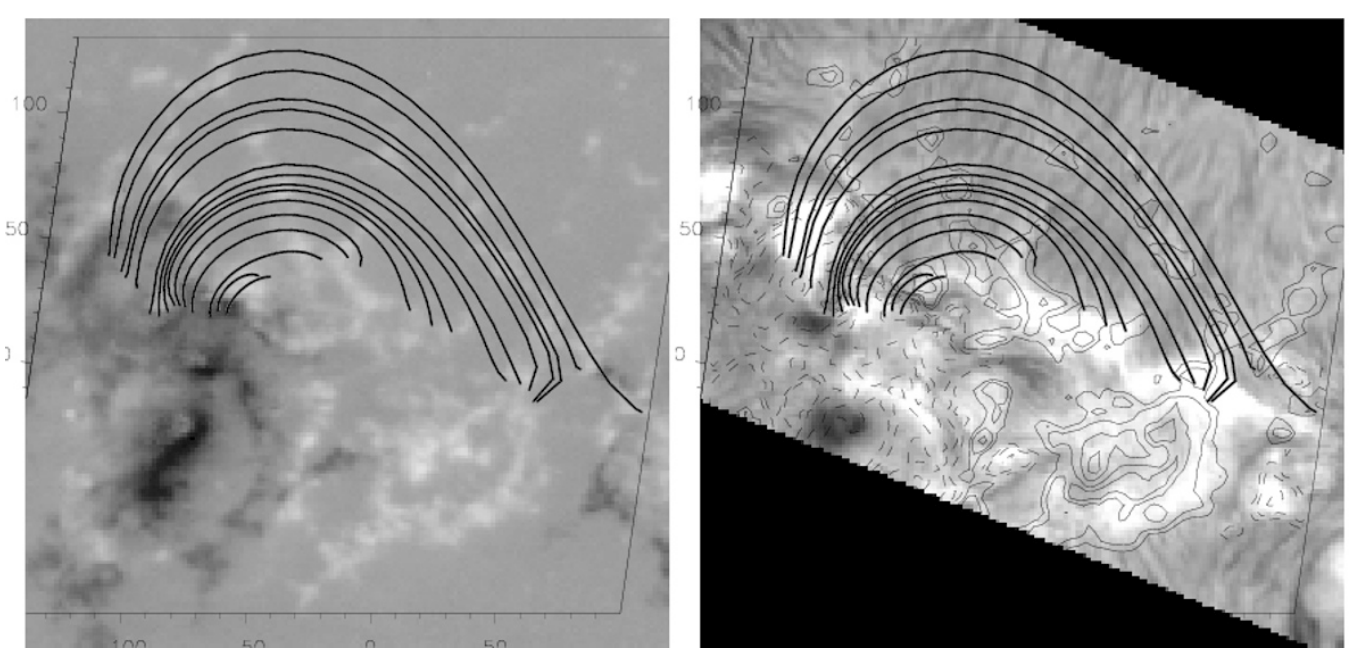

Fig. 1. Magnetic field lines extrapolated in a linear force free configuration and H $\alpha$ ribbons of the 22 October 2003 flare for two times observed with the VTT in Canaries showing the fast extension of the ribbons (Berlicki et al., 2004). 


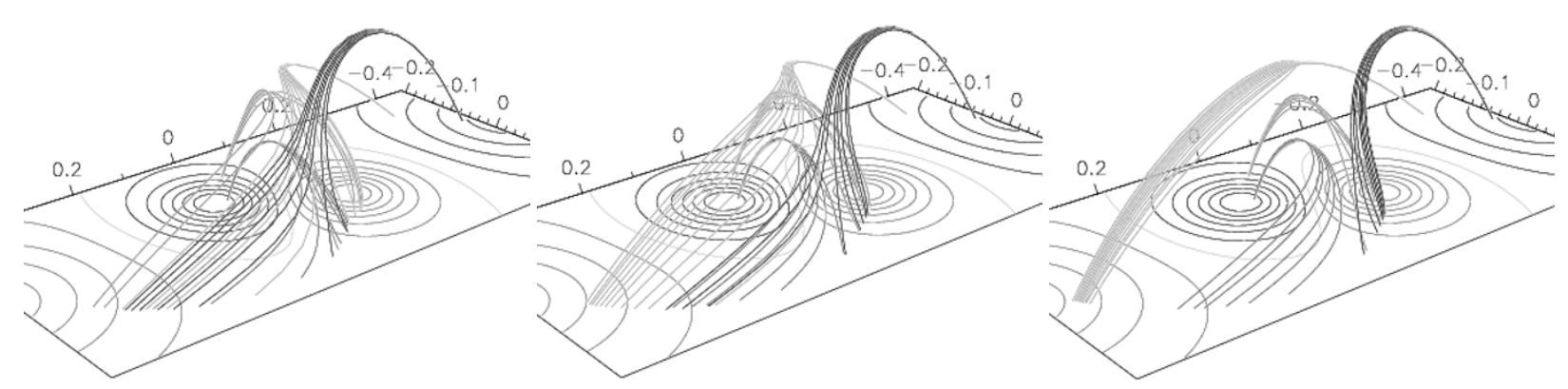

Fig. 2. Quadrupolar magnetic reconnection with slip-running field lines for three different times. The darker field lines have a fixed footpoint anchored in a fixed point in the right source, the other footpoint is slip-running continuously along the quasi-separatrice layer. During the reconnection, the plasma in the different field lines will be successively heated with footpoints visible as bright kernels. This behaviour explains the fast extension of ribbons (Aulanier et al., 2006).

\section{Auroral shapes \& dynamics due to QSL slip-running reconnection}

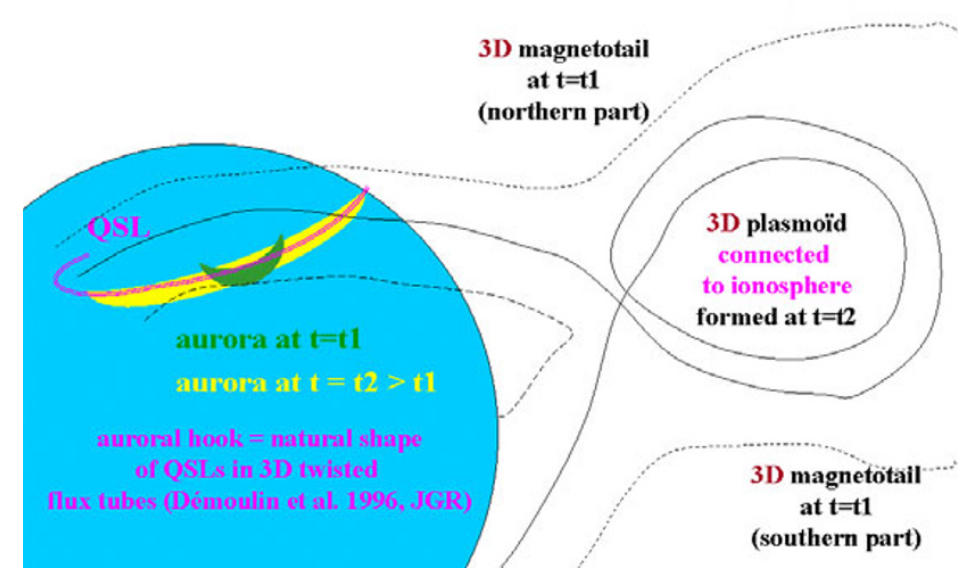

Fig. 3. Sketch of the formation of aurora due to magnetic reconnection in a QSL configuration.

short and stays short, the other one is developing an extension in a few minutes. The conclusion of the Berlicki et al. (2004)'s paper was that there was a series of reconnections in the corona. The question arises to know if this was really due to multiple reconnections. We performed a lfff extrapolation using the data base of FROMAGE and pointed out the existence of loop-like field lines growing with one fixed footpoint and the other footpoint was slip-running along the ribbon (Fig. 1).

Aulanier et al. (2006) propose a new idea with the slippage of the field lines along the QSLs (Fig. 2). Using time dependent MHD simulation it is shown the possibility of field lines to change connectivity in a continuously way and not abruptly like in the classical null point reconnection where field lines clearly connect by pair. This process can take place in many solar flares. Magnetic field configurations with fields weakly stressed by asymmetric line-tied twisting motions are considered. When the line-tied driving is suppressed, magnetic reconnection is solely due to the self-pinching and dissipation of current layers formed along QSLs. For thin QSLs and high resistivity, the field line footpoints slip-run at super-Alfvénic speeds along the intersection of the QSLs with the chromosphere. Since particles are probably accelerated in reconnection regions or in the vicinity, slip-running reconnection could explain the fast extension of the ribbons in all the wavelengths, being the result of heat of the plasma as particles travel in denser plasma. Recently Hinode observed moving loops moving that have been interpreted as due to the slippage of magnetic field lines (Aulanier et al., 2007).

\section{Reconnection in the Magnetosphere in QSLs}

Making an analogy between solar flares and magnetospheric substorms, we can conjecture that QSL reconnection may very well occur in the latter. Indeed, the geometry of the Earth magnetosphere can possibly make the appearance of real 3D null points impossible in the magneto-tail when it is stretched anti sunward. This does not preclude the development of rotational discontinuities in the tail, i.e. of $2 \mathrm{D}$ null points with an azimuthally guide field, as often reported in global models of the magnetosphere. But solar models teach us that the latter configurations can correspond to a hyperbolic flux tube (HFT), hence to QSLs when the system is viewed globally. Assuming that substorms correspond, on large scales, to the formation of a fully $3 \mathrm{D}$ twisted flux rope rooted in the ionosphere (and not a 2D plasmoid), we can transpose the solar Cartesian 3D model of Démoulin et al. (1996a) to the Earth spherical geome- 
try, replacing the solar photosphere by the ionosphere and the solar corona by the magnetosphere. This topology naturally possesses QSLs between the flux rope and the outer fields, that intersect at a HFT located between the Earth and the flux rope, and whose footprints have $J$ shapes. In this picture, polar auroras are the equivalent to chromospheric flare ribbons, i.e. they are due to the impact of particles accelerated from the reconnection site, i.e. the HFT.

Transposing our recent findings about slip running reconnection in the corona to the magnetosphere, we predict and interpret by the latter model the following evolution of aurorae: (1) the aurora starts to form a point-like brightening at the ionospheric footpoint of the HFT, where QSL reconnection is the most efficient since that is where the strongest field aligned currents (i.e. parallel electric fields) naturally develop, according to Aulanier et al. (2005); (2) the aurora brightnings expand along the footprint of the J-shaped QSLs predicted by Démoulin et al. (1996b), azimuthally in the Earth case; this fast development is due to slip-running reconnection at the HFT (as described in Aulanier et al., 2006) in the magneto-tail during the formation of the flux rope that it later ejected anti-sunward. We do not know whether these dynamics of polar aurorae are frequently observed or not, so we would like to encourage further studies to test our present transposition of solar slip-running reconnection models to magnetospheric models (Fig. 3).

\section{Conclusion}

Flares and eruptions are dynamical events which evolve with a fast speed. Past flare models were static in 2D and 3D. They provided good insight on the magnetic topology of active regions and the loci of possible deposit of energy.

We have shown that MHD simulations explain some particular aspects of the dynamics of flares. In one case we have shown how slip-running magnetic field lines in quasiseparatrice can mimic fast extension of $\mathrm{H} \alpha$ and UV ribbons observed by THEMIS and TRACE and running XRT loops observed by Hinode. Finally, making an analogy between solar flares and magnetospheric substorms, we can conjecture that QSL reconnection may very well occur in the latter.

Acknowledgments. I would like to thank Dr. Akosufu and Dr. Shibata, the organizers of the CAWSES workshop in Alaska where I present this work.

\section{References}

Antiochos, S. K., C. R. DeVore, and J. A. Klimchuk, A model for solar coronal mass ejections, Astrophys. J., 510, 485-493, 1999.

Aulanier, G., E. E. Deluca, S. K. Antiochos, A. McMullen, and L. Golub, The topology and evolution of the Bastille day flare, Astrophys. J., 540, 1126-1142, 2000.

Aulanier, G., E. Pariat, and P. Démoulin, Current sheet formation in quasiseparatrix layers and hyperbolic flux tubes, Astron. Astrophys., 444, 961-976, 2005.
Aulanier, G., E. Pariat, P. Démoulin, and DeVore, Slip-running reconnection in quasi-separatrix layers, Sol. Phys., 238, 347-376, 2006.

Aulanier, G., L. Golub, E. W. DeLuca, J. W. Cirtain, R. Kano, L. L. Lundquist, N. Nakurage, T. Sakao, and M. Weber, Science, 2007 (submitted).

Berlicki, A., B. Schmieder, N. Vilmer, G. Aulanier, and G. DeZanna, Evolution and magnetic topology of the M 1.0 flare of October 22, 2002, Astron. Astrophys., 423, 1119-1131, 2004.

Chen, P. F. and K. Shibata, An emerging flux trigger mechanism for coronal mass ejections, Astrophys. J., 545, 524-531, 2000.

DelZanna, G., B. Schmieder, H. Mason, A. Berlicki, and S. Bradshaw, The gradual phase of the X17 flare on October 28, 2003, Sol. Phys., 239, 173-191, 2006a.

DelZanna, G., A. Berlicki, B. Schmieder, and H. Mason, A multiwavelength study of the compact M1 flare on October 22, 2002, Sol. Phys., 234, 95-113, 2006b.

Démoulin, P., Magnetic topologies: where will reconnection occur?, in ESA SP , edited by Innes, D., Lagg, A., and Solanki, S. A., 596, 2005.

Démoulin, P., Extending the concept of separatrices to QSLs for magnetic reconnection, Adv. Space Res., 37, 1269-1282, 2006.

Démoulin, P., J. C. Hénoux, E. R. Priest, and C. Mandrini, QuasiSeparatrix layers in solar flares. I. Method, Astron. Astrophys., 308, 643-655, 1996a.

Démoulin, P., E. Priest, and D. Lonie, Three-dimensional magnetic reconnection without null points, J. Geophys. Res., 101, 7631-7646, 1996b.

Démoulin, P., L. G. Bagala, C. Mandrini, J. C. Hénoux, and M. G. Rovira, Quasi-separatrix layers in solar flares. II. Observed magnetic configurations, Astron. Astrophys., 325, 305-317 1997.

Forbes, T. G. and P. A. Isenberg, A catastrophe mechanism for coronal mass ejections, ApJ, 373, 294-307, 1991.

Klimchuk, J., Models of CMEs, Geographical Monograph, 125, 143, 2001.

Li, H., B. Schmieder, G. Aulanier, and A. Berlicki, Is pre-eruptive null point reconnection required for triggering eruptions?, Sol. Phys., 237, 85-100, 2006.

Lin, J., T. G. Forbes, P. Isenberg, and P. Démoulin, The effect of curvature on flux-rope models of coronal mass ejections, Astrophys. J., 504, 10061009, 1998.

Lin, J., Motions of flare ribbons and loops in various magnetic configurations, Sol. Phys., 222, 115-136, 2004.

Longcope, D. W., Topological methods for the analysis of solar magnetic fields, Living Rev. Sol. Phys., 2, 1-25, 2005.

Mandrini, C. H., P. Démoulin, B. Schmieder, E. E. DeLuca, E. Pariat, and W. Uddin, Companion event and precursor of the X17 flare on 28 October 2003, Sol. Phys., 238, 293-312, 2006.

Régnier, S. and R. C. Canfield, Evolution of magnetic fields and energetics of flares in active region 8210, Astron. Astrophys., 451, 319-330, 2007.

Schmieder, B., Magnetic source regions of coronal mass ejections, J. Astrophys. Astron., 27, 139-149, 2006.

Schmieder, B., P. Heinzel, L. van Driel, and J. R. Lemen, Post-flare loops of 26 June 1992, II, Sol. Phys.,165, 303-328, 1996.

Schmieder, B., G. Aulanier, P. Démoulin, L. van Driel, T. Roudier, N. Nitta, and G. Cauzzi, Magnetic reconnection driven by emergence of sheared magnetic field, Astron. Astrophys., 325, 1213-1225, 1997.

Schmieder, B., C. H. Mandrini, P. Démoulin, E. Pariat, A. Berlicki, and E. DeLuca, Magnetic reconfiguration before the X 17 Solar flare of October 28 2003, Adv. Space Res., 37, 1313-1316, 2006.

Schmieder, B., C. Mandrini, G. Aulanier, H. Li, and A. Berlicki, What is the role of magnetic null points in large flares?, Adv. Space Res., 39, 1840-1846, 2007.

Su, Y. N., L. Golub, A. A. van Ballegooijen, and M. Gros, Analysis of magnetic shear in an X17 solar flare on October 28, 2003, Sol. Phys., 236, 325-349, 2006.

B. Schmieder (e-mail: brigitte.schmieder@obspm.fr), G. Aulanier, P. Démoulin, and E. Pariat 